\title{
PERCEPÇÕES E CONHECIMENTOS DA EQUIPE DE ENFERMAGEM SOBRE O PROCESSO DE IMUNIZAÇÃO
}

\section{Nursing team's perceptions and knowledge of the immunization process \\ Percepciones y conocimientos del equipo de enfermería sobre el proceso de inmunización}

\author{
Roberta Farias Aragão iD \\ Universidade Federal do Ceará - UFC - Sobral (CE) - Brasil
}

Izabelle Mont'Alverne Napoleão Albuquerque (iD

Universidade Estadual Vale do Acaraú - UVA - Sobral (CE) - Brasil

Marcos Aguiar Ribeiro iD

Universidade Federal de São Paulo - UNIFESP - São Paulo (SP) - Brasil

Raissa Mont'Alverne Barreto iD

Universidade Federal do Ceará - UFC - Sobral (CE) - Brasil

Jaciara Alves de Sousa iD

Universidade Estadual Vale do Acaraú - UVA - Sobral (CE) - Brasil

\section{RESUMO}

Objetivo: Analisar as percepções, conhecimentos e atitudes da equipe de enfermagem sobre o processo de imunização. Métodos: Trata-se de um estudo exploratório, descritivo, com abordagem qualitativa, no qual se coletaram dados por meio de entrevistas semiestruturadas com 15 técnicos de enfermagem vacinadores e sete enfermeiros, no ano de 2016, no município de Sobral, Ceará, Brasil. As entrevistas abordaram questões sobre percepções, conhecimentos e atitudes da equipe de enfermagem sobre o processo de imunização, avaliando-se os dados por meio de análise temática. Resultados: Das categorias que emergiram, observou-se que a equipe de enfermagem não tem o retorno da Secretaria de Saúde em relação a importância dos indicadores coletados, de modo que faz-se necessário intensificar o processo de educação permanente da equipe em relação às possíveis situações adversas advindas da imunização. Observaram-se alguns aspectos facilitadores relacionados ao acolhimento e à implicação da equipe em orientar quanto à importância da imunização para promover a autonomia e capacitar os usuários para o autocuidado. Conclusão: Os resultados evidenciaram fatores limitantes que impactam negativamente na oferta do serviço, sendo necessária, assim, a proposição de ajustes no processo de capacitação para o favorecimento de mudanças que permitam o bom desenvolvimento das atividades em sala de vacina.

Descritores: Cobertura Vacinal; Programas de Imunização; Imunização; Atenção Primária à Saúde.

\section{ABSTRACT}

Objective: To analyze the nursing team's perceptions, knowledge and attitudes regarding the immunization process. Methods: This is a qualitative exploratory and descriptive study in which data were collected through semi-structured interviews with 15 vaccinating nursing technicians and seven nurses in 2016 in Sobral, Ceará, Brazil. The interviews contained questions about the nursing team's perceptions, knowledge and attitudes regarding the immunization process and the data underwent thematic analysis. Results: The categories that emerged showed that the nursing team does not get feedback from the Health Secretariat regarding the importance of the measured indicators; therefore, it is necessary to intensify the permanent training of the team with regard to possible adverse situations resulting from immunization. There were some facilitating aspects related to user embracement by the team and its commitment to advise on the importance of immunization to promote autonomy and empower users for self-care. Conclusion: The results showed limiting factors that negatively impact the service provision. Thus, it is necessary to propose adjustments in the training process to favor changes that allow the good development of activities in the vaccine room.

Descriptors: Immunization Coverage; Immunization Programs; Mass Vaccination; Primary Health Care.

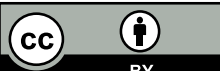




\section{RESUMEN}

Objetivo: Analizar las percepciones, los conocimientos y las actitudes del equipo de enfermería sobre el proceso de inmunización. Métodos: Se trata de un estudio exploratorio, descriptivo y de abordaje cualitativo en el cual se recogieron los datos a través de entrevistas semiestructuradas con 15 técnicos de enfermería que aplican vacunas y siete enfermeros en el año 2016 en el municipio de Sobral, Ceará, Brasil. Las entrevistas abordaron cuestiones sobre las percepciones, los conocimientos y las actitudes del equipo de enfermería sobre el proceso de inmunización y los datos han sido evaluados a través del análisis temático. Resultados: De las categorías que emergieron se observó que el equipo de enfermería no tiene la devolución de la Secretaria de Salud respecto la importancia de los indicadores recogidos de manera que se hace necesario la intensificación del proceso de educación permanente del equipo sobre las posibles situaciones adversas de la inmunización. Se observaron algunos aspectos facilitadores relacionados a la acogida y la implicación del equipo para orientar sobre la importancia de la inmunización para la promoción de la autonomía y capacitación de los usuarios sobre al autocuidado. Conclusión: Los resultados evidenciaron los factores limitantes que impactan de manera negativa para la oferta del servicio con la necesidad de proponer ajustes para el proceso de capacitación que favorezca los cambios que permitan el desarrollo adecuado de las actividades de vacunación.

Descriptores: Cobertura de Vacunación; Programas de Inmunización; Inmunización; Atención Primaria de Salud.

\section{INTRODUÇÃO}

O Programa Nacional de Imunizações (PNI), criado em 1973, é responsável pela organização da Política Nacional de Vacinação, a qual tem como missão o controle, a erradicação e a eliminação das doenças imunopreveníveis ${ }^{(1)}$. A política fortaleceu os municípios por meio da descentralização da gestão dos serviços e ações em saúde em relação à imunização, de maneira que a Atenção Primária à Saúde (APS) tornou-se responsável por todo o processo de administração dessas atividades ${ }^{(2)}$.

Todavia, a literatura científica mostra importantes deficiências nas salas de vacina (SV), principalmente no que se refere à conservação dos imunobiológicos e à adesão às normas e diretrizes estabelecidas pelo $\mathrm{PNI}$, bem como aspectos restritivos relacionados à supervisão das SV, o que pode comprometer a efetividade do PNI e qualidade dos imunobiológicos disponibilizados, haja vista que a supervisão configura-se como um instrumento de avaliação da qualidade da assistência prestada à população ${ }^{(3,4)}$. Além disso, o Ministério da Saúde (MS) preconiza que essa supervisão ocorra sistematicamente, de forma a verificar as condições da área física e o cumprimento de normas que visam a garantir a qualidade dos imunobiológicos, desde sua fabricação e conservação adequada até a aplicação(1).

Assim, para o favorecimento e manutenção da qualidade dos serviços de imunização, é necessário compreender todo o processo que envolve uma vacinação eficaz, visto que esta é a principal estratégia de prevenção das doenças imunopreveníveis e promoção da saúde ${ }^{(5)}$.

Dada a relevância e o impacto indiscutível que a imunização exerce na saúde das populações, torna-se fundamental a condução de estudos que visam ao conhecimento e análise dos serviços de imunização para implementação de práticas seguras que subsidiam o alcance das metas do PNI.

Nessa perspectiva, o objetivo deste estudo foi analisar as percepções, conhecimentos e atitudes da equipe de enfermagem sobre o processo de imunização.

Nesse ínterim, considera-se relevante este estudo por acreditar que a análise contínua dos processos de trabalho possibilita a reflexão e orienta profissionais e gestores de saúde no tocante à organização dos serviços para redirecionamento de suas ações. Assim, para gerenciar efetivamente um programa, é necessário conhecer como estão sendo produzidos esses serviços e quais as condições dadas para sua real efetivação.

\section{MÉTODOS}

Trata-se de uma pesquisa exploratória, descritiva, com abordagem qualitativa, a qual permite compreender as informações e conhecimentos quanto ao objeto de pesquisa, trabalhando com o universo dos significados, dos motivos, das aspirações, dos valores, das crenças e das atitudes, inerentes à realidade social ${ }^{(6)}$.

Desenvolvido no ano de 2016, este estudo teve como cenário o município de Sobral, Ceará, Brasil, localizado no semiárido cearense. Participaram do estudo 15 técnicos de enfermagem vacinadores e sete enfermeiros que atuam em SV de Unidades Básicas de Saúde (UBS). Considerou-se como critério de inclusão: ter experiência profissional de pelo menos seis meses em SV. Estabeleceu-se como critério de exclusão: estar de férias, licença médica ou afastamento por qualquer natureza no período da coleta de dados. 
Utilizou-se como técnica de coleta de dados a realização de entrevistas semiestruturadas para análise de percepções, conhecimentos e atitudes da equipe sobre o processo de imunização do referido município. Desse modo, agendou-se as entrevistas previamente, em data e horário escolhidos pelos participantes, assim como o local de realização. Registraram-se as falas para posterior transcrição e minuciosa análise. Utilizou-se, também, um diário de campo com anotações de acontecimentos pertinentes desde o planejamento até a execução das entrevistas.

Vale salientar ainda que, após a constatação de que os dados coletados eram suficientes para atender ao método e ao objetivo proposto, encerrou-se a coleta de dados, tendo por base o princípio da saturação teórica.

O exame das informações apreendidas ocorreu por meio da análise temática(6) ${ }^{(6}$ seguindo as fases de organização dos dados, de modo a considerar: o objetivo do estudo; a exploração do material empírico, com leitura exaustiva dos registros; a separação dos recortes de acordo com as convergências e divergências; e a classificação e agregação dos dados em temas.

Para garantia do anonimato dos participantes e a sistematização dos discursos analisados, identificou-se os técnicos de enfermagem com a letra $V$, correspondendo a vacinador, e os enfermeiros, com a letra $E$, seguida de números sequenciais de 1 a 15 para os vacinadores e de 1 a 7 para as enfermeiras.

A pesquisa apresentou parecer favorável da Comissão Científica da Secretaria Municipal de Saúde de Sobral e do Comitê de Ética em Pesquisas da Universidade Estadual Vale do Acaraú (UVA) de acordo com a Resolução 466/2012 sob o Parecer n. ${ }^{\circ} 1.143 .315$.

\section{RESULTADOS E DISCUSSÃO}

Atendendo ao processo de imersão do estudo, a partir dos resultados inferidos nas informações coletadas, emergiram três categorias temáticas para analisar: conhecimentos e atitudes dos profissionais sobre os indicadores de imunização e as situações adversas, além do acolhimento e educação em saúde em imunização, as quais serão discorridas a seguir.

\section{Conhecimentos e atitudes sobre indicadores de imunização}

Verificou-se que as taxas de coberturas vacinais e taxas de abandono do território são completamente desconhecidas pelos profissionais do serviço de imunização. Nenhum dos entrevistados demonstrou conhecimento acerca dos indicadores de imunização em seus territórios. A centralização das informações na Secretaria de Saúde/ PNI foi observada nas falas dos participantes:

"Cobertura vacinal mesmo eu não sei, isso fica lá na secretaria. A gente procura atingir a demanda e, quando a demanda não vem, o ACS vai procurar. Todo fim do mês eu vejo quais foram as pessoas que não vieram e passo pra enfermeira, para o ACS fazer a busca ativa." (V8)

Um resultado semelhante foi encontrado em um estudo realizado em Belo Horizonte, em que foi possível observar que os profissionais entrevistados não valorizam a monitoração da cobertura vacinal como uma estratégia para gestão e programação das ações em saúde da UBS. No entanto ressalta-se que a adequada gestão da informação está ligada à cultura institucional de valorização da informação, ou seja, do sentimento de participação e responsabilidade de produção e utilização da informação( ${ }^{(7)}$.

Nesse contexto, o indicador de cobertura vacinal representa um importante instrumento para a tomada de decisão nas diferentes esferas de gestão, visto que somente com coberturas adequadas é possível alcançar o controle, ou manter em condição de eliminação ou erradicação as doenças imunopreveníveis sob vigilância. Além de adequadas, as coberturas de vacinação precisam ser homogêneas, uma vez que esse fator é um importante indicador de desempenho do $\mathrm{PNI}^{(8)}$.

No que se refere às ações para obter maior cobertura vacinal e menor taxa de abandono no território, os entrevistados reportam atos comuns de análise da situação vacinal, especialmente das crianças, às equipes de saúde:

"Todo fim de mês tem o encontro da enfermeira com os ACS. Elas fazem um levantamento e busca ativa se tiver atrasado." (V3)

"Geralmente, as ACS vão avisar e chamam quando está atrasada. Se a mãe não vier, a enfermeira faz uma visita. E, na triagem de manhã, a enfermeira fala das vacinas." (V12)

Diante dos relatos, constata-se interesse e preocupação dos vacinadores em orientar os usuários quanto à importância da imunização e o aprazamento de vacinas subsequentes. Assim, entende-se que todos os profissionais atuantes em unidades de saúde devem estar preparados para atuar como educadores em vacinação, envolvendo 
mais do que apenas o contato com enfermeiros ou médicos, de modo que qualquer contato com um funcionário da unidade de saúde torne-se uma oportunidade para se abordar a situação vacinal do usuário( ${ }^{(9)}$.

Estudos revelam determinadas estratégias de intervenção, tais como educação em saúde e visitas domiciliares visando a abordar os usuários podem aumentar a cobertura de vacinas. Discussão baseada em evidências que visa à tradução de conhecimento para os membros da comunidade por profissionais de saúde pode ser mais eficaz do que as estratégias de informação convencionais ${ }^{(10,11)}$.

Nessa perspectiva, a análise da situação vacinal efetuada com informações coletadas por meio de visitas domiciliares dos ACS traz dados que, além de ajudar a conhecer melhor os indicadores de saúde da população, remete a questões relativas às relações dos profissionais de saúde com os usuários. Além disso, as consultas de puericultura tornam-se importantes aliadas neste repasse de informações, uma vez que os dados relativos à ausência das crianças nas consultas de puericultura evidenciam uma maior chance para atrasos em suas vacinas ${ }^{(12)}$.

\section{Conhecimentos e atitudes frente às situações adversas de imunização}

É conhecido que as vacinas podem causar eventos adversos após sua aplicação. Contudo, os riscos de complicações graves relacionados são muito menores do que os das doenças contra as quais elas protegem. É preciso ter em mente que a confiança do público nas vacinas ofertadas é condição-chave para o sucesso do PNI e que as vacinas hoje disponíveis são consideradas seguras, mas não livres de risco ocasional( ${ }^{(8)}$.

Diante dos relatos dos profissionais de saúde, notou-se um conhecimento superficial, tanto das medidas a serem tomadas em casos de eventos adversos quanto da existência do Manual dos Centros de Referência para Imunobiológicos Especiais (CRIE):

"Quando a criança vem fazer a vacina, a mãe é informada da reação que pode acontecer. Caso venha a acontecer, a criança vai ser encaminhada ao hospital e depois ela retorna ao posto e ali é notificada a reação pela enfermeira. A notificação é encaminhada para a Secretaria de Saúde e lá eles encaminham pra Fortaleza pra poder vir a vacina que substitui aquela que fez a reação." (V4)

Além disso, destacaram-se dificuldades dos vacinadores em executar práticas de saúde de qualidade, em virtude de poucos treinamentos que lhes são ofertados:

"Às vezes, fico com muitas dúvidas na hora de vacinar, porque o calendário muda muito e tenho que ir até o enfermeiro para ter certeza. Se a gente tivesse mais momentos sobre as atualizações, acho que facilitaria nossa situação." (V3)

Outros estudos também destacam a falta de preparo dos profissionais vacinadores em relação às suas atividades na sala de vacina e enfatizam essa deficiência como um grande desafio nos serviços de saúde, visto que as inadequadas práticas de vacinação podem causar danos aos usuários e comprometer a qualidade e credibilidade dos imunobiológicos ${ }^{(13,14)}$.

A partir disso, é notório que a temática em questão sugere a atualização de conhecimentos e aquisição de novas informações, remetendo ao processo de educação continuada. No entanto, na perspectiva de transformação da prática, deve-se desenvolver no profissional de saúde uma consciência crítica, potencializada pela aquisição de novos conhecimentos, conquistada por meio das mudanças de atitudes decorrentes das experiências vividas e de sua transformação pessoal, profissional e social, traduzindo-se em educação permanente ${ }^{(9)}$.

Para isso, é importante salientar que a gestão e o enfermeiro responsável pela sala de vacinação devem oferecer suporte e apoio nesses processos de capacitação contínua dos vacinadores. O que se percebe, porém, a partir dos resultados deste estudo e de outros na literatura científica, é que os treinamentos não acontecem na mesma proporção das mudanças ocorridas no calendário nacional ${ }^{(15,16)}$.

Dessa forma, se faz necessário que a gestão conheça as principais barreiras enfrentadas no cotidiano pelos profissionais na sala de vacinação para refletir estratégias de aperfeiçoamento e transformações das práticas para que ocorram melhorias no processo de trabalho em sala de vacina.

Os discursos dos participantes desta pesquisa resumem-se à execução de tarefas repassadas pela Vigilância Epidemiológica, em que se percebeu a não valorização dos profissionais quanto à realização de atividades de educação em saúde junto à comunidade, limitando-se a realizar o bloqueio para quebra da cadeia de transmissão em situação de doença imunoprevenível no território, como pode ser evidenciado a seguir:

"Só teve sarampo e a gente fez o bloqueio nos quarteirões vizinhos. A única forma de prevenir é o bloqueio, e acompanhamos o paciente, fazemos a sorologia e orientamos quanto ao tratamento e a prevenção." (E6) 
Os resultados de um estudo sobre as percepções de usuários frente ao acesso à vacinação revelaram que a falta de conhecimento deles sobre imunização surgiu como fator que compromete o acesso a esse serviço. Embora tenha sido cada vez mais evidenciado o uso da mídia como fonte de informação sobre medidas preventivas, há a necessidade de fortalecimento das ações de educação em saúde para que alcancem os diferentes estratos sociais. Os autores enfatizam, ainda, que o processo de aprendizado contínuo provoca novas concepções e atitudes na recriação da própria maneira de ser e de se cuidar. Além disso, a informação e comunicação efetiva são essenciais para que se alcance a medida preventiva da vacinação como protetora da saúde individual e coletiva ${ }^{(17)}$.

\section{Acolhimento e educação em saúde em imunização}

De acordo com os entrevistados, todos os funcionários da UBS recebem informações sobre a importância da vacinação e encaminham a clientela à SV, apesar de não terem conhecimento específico sobre as vacinas disponíveis. As falas contemplam essa realidade:

"Do vigia a copeira, todo mundo que procurar vacina, eles sabem dirigir a pessoa até a sala. Sabem acolher." (V6)

"Nas rodas, a gerente sempre bate na mesma tecla: temos que dar as informações corretas. Todos são responsáveis pela saúde da comunidade." (E7)

Nesta pesquisa, identificou-se que profissionais de outras categorias encaminham o usuário para a SV, sendo considerada uma atitude significativa para o aumento da cobertura vacinal. Uma das causas de baixa cobertura vacinal é a perda de oportunidades para vacinar. Assim, a sensibilização dos profissionais da equipe para que se envolvam na atividade de vacinação constitui-se como estratégia fundamental para superação desse entrave(18).

Os resultados do presente estudo mostraram que as ações de educação em saúde referentes à imunização são praticadas em momentos pontuais de atendimentos de puericultura, acolhimento e triagem, grupos de convivência e períodos pré-campanha de vacinação. Apesar disso, os discursos não demonstram intensidade, e as estratégias são limitadas na maioria das UBS analisadas:

"No dia da puericultura a gente fala... Também tem o dia do HIPERDIA, que tem os idosos e os adultos. Nos grupos que temos aqui, sempre falamos sobre vacinas, especialmente pré-natal e puericultura." (E4)

"É mais pelos agentes de saúde... Às vezes, vamos pra rádio falar da campanha... Infelizmente, não fazemos muita ação coletiva, estamos falhando nisso." (E3)

Assim, observa-se que esses profissionais mantêm a dicotomia entre a prática de procedimentos técnicos de enfermagem e a educação em saúde, as quais deveriam estar integradas durante a execução do processo de imunização. Dessa maneira, tal fato pode conduzir os usuários ao descumprimento da vacinação periódica e evadir o processo de vacinação. É importante salientar, porém, que, no contexto da saúde, a prática educativa voltada aos usuários é considerada uma tecnologia para a concretização do SUS, visto que permite discussões e recomendações que possibilitam aumentar a capacidade no autocuidado à saúde ${ }^{(19)}$.

Há reflexão sobre a importância da incorporação de novas tecnologias educativas no processo de educação em saúde na atualidade, as quais contribuem para democratização da comunicação, da educação e do conhecimento a partir da realidade cultural dos indivíduos. O uso de técnicas audiovisuais tem se mostrado crescente nas pesquisas experimentais nos últimos tempos. Esse recurso permite que se repensem estratégias de cuidado, fazendo parte, inclusive, do processo terapêutico em muitas abordagens ${ }^{(20)}$.

Assim, acredita-se que o investimento em tecnologias e estratégias educativas de imunização potencializa o serviço e facilita o acesso, de forma a reforçar os conceitos de uma nova forma de fazer saúde que pode transformar uma realidade e contribuir para a melhoria da qualidade da assistência em saúde ${ }^{(21)}$.

Por fim, os resultados apresentados evidenciam a necessidade de maior valorização e fortalecimento das atividades educativas pelos profissionais, além de suporte que forneça subsídios para a proposição de ajustes no processo de capacitação e, com isso, no favorecimento de mudanças que permitam o bom desenvolvimento das atividades em sala de vacina.

\section{CONSIDERAÇÕES FINAIS}

A partir da realização deste estudo, identificaram-se aspectos restritivos no que se refere ao desenvolvimento de ações pontuais de educação em saúde, ao desconhecimento dos dados e indicadores do serviço, e às situações 
adversas à imunização. Além disso, a partir dos discursos, verificaram-se aspectos facilitadores no que concerne ao acolhimento dos usuários pela equipe de saúde e sensibilização destes em orientar os usuários quanto à importância da imunização, em diversas atividades e momentos realizados nas Unidades Básicas de Saúde, com o intuito de promover a autonomia e capacitar os usuários para o autocuidado.

Dessa forma, verifica-se que esses aspectos restritivos implicam na oferta de um serviço regular, mas com potencial para alcançar uma qualificação desejada a partir do fortalecimento contínuo da educação permanente da equipe de enfermagem que atua em salas de vacina.

Finalmente, reconhecendo a importância da imunização como forte instrumento de proteção à saúde, torna-se fundamental a continuidade de estudos para aprofundamento de questões que permitam solucionar as lacunas encontradas neste estudo, a fim de assegurar a qualidade das vacinas disponibilizadas à população e atingir as metas estabelecidas pelo Programa Nacional de Imunizações.

\section{CONFLITOS DE INTERESSE}

Os autores declaram que não houve conflitos de interesses na concepção deste trabalho.

\section{CONTRIBUIÇÕES}

Roberta Farias Aragão, Izabelle Mont'Alverne Napoleão Albuquerque e Marcos Aguiar Ribeiro contribuíram com a concepção e delineamento; a análise e interpretação dos dados; a redação do artigo; a revisão crítica e a aprovação da versão a ser publicada. Raissa Mont'Alverne Barreto e Jaciara Alves de Sousa contribuíram com a redação do artigo e a aprovação da versão a ser publicada.

\section{REFERÊNCIAS}

1. Ministério da Saúde (BR). Secretaria de Vigilância em Saúde. Departamento de Vigilância de Doenças Transmissíveis. Manual de Normas e Procedimentos para Vacinação. Brasília: ministério da saúde; 2014.

2. Oliveira VC, Gallardo MDPS, Arcêncio RA, Gontijo TL, Pinto IC. Avaliação da qualidade de conservação de vacinas na Atenção Primária à Saúde. Cien Saúde Colet [Internet]. 2014 [acesso em 2019 Abr 26];19(9):3889-98. Disponível em: http://www.scielo.br/scielo.php?pid=S141381232014000903889\&script=sci_abstract\&tIng=pt

3. Luna GLM, Vieira LJES, Souza PF, Lira SVG, Moreira DP, Pereira AS. Aspectos relacionados à administração e conservação de vacinas em centros de saúde no Nordeste do Brasil. Cien Saúde Colet [Internet]. 2011 [acesso em 2018 Maio 15];16(2):513-21. Disponível em: http://www.scielo.br/scielo.php?script=sci_arttext\&pi $\mathrm{d}=\mathrm{S} 1413-81232011000200014$

4. Oliveira VC, Gallardo PS, Gomes TS, Passos LMR, Pinto IC. Supervisão de enfermagem em sala de vacina: a percepção do enfermeiro. Texto \& Contexto Enferm [Internet]. 2013 [acesso em 2019 Abr 27];22(4):101521. Disponível em: http://www.scielo.br/scielo.php?script=sci_arttext\&pid=S010407072013000400018\&lng= en. http://dx.doi.org/10.1590/S0104-07072013000400018.

5. Cunha JO, Oliveira IMB, Santos AD, Cunha MWN, Santos FJ, Santos JMJ. Avaliação da padronização dos procedimentos nas salas públicas de vacinas do município de Itabaiana, Sergipe, Brasil. Rev Bras Pesqui Saúde [Internet]. 2018 [acesso em 2019 Jun 15];20(1):70-8. Disponível em: http://periodicos.ufes.br/RBPS/ article/view/20610/13855.https://doi.org/10.21722/rbps.v20i1.20610

6. Minayo MCS. O desafio do conhecimento: pesquisa qualitativa em saúde. 12a ed. São Paulo: Hucitec; 2010.

7. Lages AS, França EB, Freitas MIF. Profissionais de saúde no processo de vacinação contra hepatite $B$ em duas unidades básicas de Belo Horizonte: uma avaliação qualitativa. Rev Bras Epidemiol [Internet]. 2013 [acesso em 2018 Out 28];16(2):364-75. Disponível em: http://www.scielo.br/scielo.php?pid=S1415790X2013000200364\&script=sci_abstract\&tlng=pt

8. Ministério da Saúde (BR). Coberturas Vacinais no Brasil: Período: 2010 - 2014. Brasília: Ministério da Saúde; 2015. 
9. Assad SGB, Corvino MPF, Santos SCP, Cortez EA, Souza FL. Educação permanente em saúde e atividades de vacinação: revisão integrativa. Rev Enferm UFPE [Internet]. 2017 [acesso em 2018 Out 28];11(Supl. 1):410-21. Disponível em: https://periodicos.ufpe.br/revistas/revistaenfermagem/article/view/11922

10. Oyo-Ita A, Wiysonge CS, Oringanje C, Nwachukwu CE, Oduwole O, Meremikwu MM. Interventions for improving coverage of child immunisation in low- and middle-income countries. Cochrane Database Syst Rev [Internet]. 2016 [acesso em 2018 Out 22];10;7:CD008145. doi: 10.1002/14651858.CD008145.pub3. Disponível em: https://www.ncbi.nlm.nih.gov/pubmed/27394698

11. Esposito S, Principi N, Cornaglia G. Barriers to the vaccination of children and adolescents and possible solutions. Clin Microbiol Infect [Internet]. 2014 [acesso em 2015 Set 04];20(5):25-31. Disponível em: https:// www.ncbi.nlm.nih.gov/pubmed/24354949.

12. Fernandes ACN, Gomes KRO, Araújo TME, Moreira-araújo RSR. Análise da situação vacinal de crianças pré-escolares em Teresina (PI). Rev Bras Epidemiol [Internet]. 2015 [acesso em 2018 Out 30];18(4):870-82. Disponível em: http://www.scielo.br/scielo.php?pid=\$1415790X2015000400870\&script=sci_abstract\&tlng=pt.

13. Silva SS, Oliveira VC, Ribeiro HCTC, Alves TGS, Cavalcante RB, Guimarães EAA. Análise dos eventos adversos após aplicação de vacinas em Minas Gerais, 2011: um estudo transversal. Epidemiol Serv Saude [Internet]. 2016 [acesso em 2019 Jun 15];25(1):45-54. Disponível em: http://www.scielo.br/scielo. php?script=sci_arttext\&pid=S2237-96222016000100045.

14. Santos CAPS, Costa RS, Silva JLM, Santos MRF, Gomes BLF. Conhecimento, atitude e prática dos vacinadores sobre vacinação infantil em Teresina-PI, 2015. Epidemiol Serv Saude [Internet]. 2017 [acesso em 2018 Out 29];26(1):133-40. Disponível em: http://www.scielo.br/scielo.php?pid=S223796222017000100133\&script=sci_abstract\&tIng=pt.

15. Ferreira AV, Freitas PHB, Viegas SMF, Oliveira VC. Acesso à sala de vacinas da estratégia saúde da família: aspectos organizacionais. Rev Enferm UFPE [Internet]. 2017 [acesso em 2019 Jun 15];11(10):3869-77. Disponível em: http://bases.bireme.br/cgi-bin/wxislind.exe/iah/online/?/sisScript=iah/iah.xis\&src=google\&base $=$ BDENF\&lang=p\&nextAction=Ink\&exprSearch=33061\&indexSearch=ID.

16. Oliveira VC, Rennó HMS, Santos YR, Rabelo AFG, Gallardo MDPS, Pinto IC. Educação para o trabalho em sala de vacina: percepção dos profissionais de enfermagem. Rev Enferm Cent-Oeste Min [Internet]. 2016 [acesso em 2018 Out 28];6(3):2331-41. Disponível em: http://www.seer.ufsj.edu.br/index.php/recom/article/ view/1180

17. Duarte DC, Oliveira VC, Guimarães EAA, Viegas SMF. Acesso à vacinação na Atenção Primária na voz do usuário: sentidos e sentimentos frente ao atendimento. Esc Anna Nery Rev Enferm [Internet]. 2019 [acesso em 2019 Jun 16];23(1):e20180250. Disponível em: http://www.scielo.br/scielo.php?script=sci_ arttext\&pid=S1414-81452019000100203\&Ing=en\&nrm=iso\&tlng=pt

18. Tertuliano GC, Stein AT. Atraso vacinal e seus determinantes: um estudo em localidade atendida pela Estratégia Saúde Família. Cien Saúde Colet [Internet]. 2011 [acesso em 2019 Jun 15];16(2):523-30. Disponível em: http://www.scielo.br/scielo.php?script=sci_abstract\&pid=\$1413-81232011000200015\&lng=en \&nrm=iso\&tlng=pt

19. Moutinho CB, Almeida ER, Leite MTS, Vieira MA. Dificuldades, desafios e superações sobre educação em saúde na visão de enfermeiros de saúde da família. Trab Educ Saúde [Internet]. 2014 [acesso em 2018 Out 28];12(2):253-72. Disponível em: http://www.scielo.br/scielo.php?pid=S1981$77462014000200003 \&$ script=sci_abstract\&tIng=pt

20. Interaminense INCS, Oliveira SC, Leal LP, Linhares FMP, Pontes CM. Tecnologias educativas para promoção da vacinação contra o papiloma vírus humano: Revisão integrativa da literatura. Texto \& Contexto Enferm [Internet]. 2016 [acesso em 2019 Jun 17];25(2):e2300015. Disponível em: http://www.scielo.br/pdf/tce/ v25n2/0104-0707-tce-25-02-2300015.pdf

21. Oliveira VG, Pedrosa KKA, Monteiro AI, Santos ADB. Vacinação: o Fazer da Enfermagem e o saber das mães e/ou cuidadores. Rev Rene [Internet]. 2010 [acesso em 2019 Jun 18];11:133-41. Disponível em: http:// www.repositorio.ufc.br/bitstream/riufc/12577/1/2010_art_vgoliveira.pdf 


\section{Endereço para correspondência:}

Roberta Farias Aragão

Universidade Federal do Ceará - UFC

Rua Cel. Estanislau Frota, 563

Bairro: Centro

CEP: 62010-560 - Sobral - CE

E-mail: robertaaragao@hotmail.com

Como citar: Aragão RF, Albuquerque IMN, Ribeiro MA, Barreto RM, Sousa JA. Percepções e conhecimentos da equipe de enfermagem sobre o processo de imunização. Rev Bras Promoç Saúde. 2019;32:8809. 